\title{
DEGRADAÇÃO DO EFLUENTE GERADO NA ETAPA DE TINGIMENTO DE PELES DE PEIXE POR FOTOCATÁLISE HETEROGÊNEA
}

\author{
M. V. S. RIBEIRO ${ }^{1}$, V. SLUSARSKI-SANTANA ${ }^{1}$ e N. R. C. FERNANDES-MACHADO ${ }^{2}$ \\ ${ }^{1}$ Universidade Estadual do Oeste do Paraná, Centro de Engenharias e Ciências Exatas \\ ${ }^{2}$ Universidade Estadual de Maringá, Departamento de Engenharia Química \\ E-mail para contato: maycon.senna.eq@gmail.com
}

\begin{abstract}
RESUMO - O objetivo deste trabalho foi avaliar a degradação do efluente de tingimento de peles de peixe por fotocatálise empregando catalisadores suportados. Foram preparados e caracterizados catalisadores de 5 e $10 \%$ de $\mathrm{ZnO}$ suportados em zeólita A, assim como catalisadores mistos suportados $(0,1 \%$ de $\mathrm{Fe}_{2} \mathrm{O}_{3}$ ou $0,1 \%$ de $\mathrm{Nb}_{2} \mathrm{O}_{5}$ e $5 \%$ de $\mathrm{ZnO}$ ). Os testes fotocatalíticos tiveram duração de $3 \mathrm{~h}$ e foram testadas diferentes massas de catalisador $\left(1 \mathrm{e} 2 \mathrm{gL}^{-1}\right)$. A eficiência do processo foi avaliada em termos de condutividade, turbidez e descoloração. Os resultados mostraram que os catalisadores apresentam características estruturais e eletrônicas semelhantes, cristalinidade e atividade. Os testes fotocatalíticos mostraram que o aumento da massa de catalisador e do teor de $\mathrm{ZnO}$ apresentaram efeito positivo, e que a mistura dos óxidos apresentou efeito sinérgico positivo em comparação ao $5 \% \mathrm{ZnO} / \mathrm{A}$, aumentando a eficiência do processo em termos de aumento da condutividade. $\mathrm{O}$ processo fotocatalítico ocasionou redução significativa da turbidez, principalmente para o 10\% $\mathrm{ZnO} / \mathrm{A}$. Os melhores resultados de descoloração foram obtidos com $2 \mathrm{gL}^{-1}$ de zeólita A.
\end{abstract}

\section{INTRODUÇÃO}

A utilização de couro de peixes mostra-se como uma alternativa de reaproveitamento de resíduos em um cenário de intensificação da piscicultura, devido à demanda do filé de tilápia (Rebouças et al., 2011). O processo de curtimento abrange etapas de lavagem, em que grandes volumes de efluentes são gerados (Maluf, 2010). Nesse contexto, surgem problemas relacionados ao descarte de efluentes ricos em corantes reativos (Marin et al., 2014).

Dentre os processos de tratamento de efluentes, a fotocatálise está sendo muito utilizada na degradação de efluentes industriais e domésticos (Lima et al., 2010) e baseia-se na irradiação de um semicondutor, formando sítios oxidantes e redutores, catalisando reações químicas e oxidando compostos orgânicos (Peternela e Santana, 2013). Vários são os semicondutores utilizados $\left(\mathrm{TiO}_{2}, \mathrm{ZnO}, \mathrm{CdS}\right.$ e $\left.\mathrm{Fe}_{2} \mathrm{O}_{3}\right)$, os quais podem ser utilizados separados ou combinados. $\mathrm{O} \mathrm{TiO}_{2}$ é o mais usado tanto em suspensão quanto imobilizado, entretanto o $\mathrm{ZnO}$ surge como alternativa, por apresentar características semelhante à do $\mathrm{TiO}_{2}$, como por exemplo a energia de band gap (Feltrin, 2010). 
As zeólitas compõem uma classe de materiais de baixo custo, acessíveis, excelentes trocadores iônicos com grande área superficial, utilizadas tanto em processos de adsorção como suporte para fotocatalisadores, aumentando sua atividade fotocatalítica, evitando sinterizações e facilidade de filtração da solução tratada (Merg et al., 2010). Desta forma, o presente trabalho teve por objetivo avaliar a degradação do efluente do tingimento de peles de peixes utilizando fotocatalisadores suportados. Zéolita A comercial foi utilizada como suporte para imobilizar diferentes teores de $\mathrm{ZnO}$ puro e óxidos mistos $\left(\mathrm{Nb}_{2} \mathrm{O}_{5}-\mathrm{ZnO}\right.$ e $\left.\mathrm{Fe}_{2} \mathrm{O}_{3}-\mathrm{ZnO}\right)$, de modo a avaliar as melhores condições experimentais para a degradação deste efluente.

\section{MATERIAL E MÉTODOS}

\subsection{Preparo dos Fotocatalisadores}

As soluções precursoras de $\mathrm{ZnO}$ e $\mathrm{Fe}_{2} \mathrm{O}_{3}$ foram preparadas a partir da solubilização de nitrato de zinco (Química Fina) em água desionizada, em quantidade suficiente para preparar catalisadores com 5 e 10\% (\% mássica) de $\mathrm{ZnO}$, e nitrato de ferro III em água desionizada para obter o teor de $0,1 \%$ (\% mássica) de $\mathrm{Fe}_{2} \mathrm{O}_{3}$. A solução precursora de $\mathrm{Nb}_{2} \mathrm{O}_{5}$ foi obtida pela solubilização do ácido nióbico (HY-340 da CBMM com 80\% de $\mathrm{Nb}_{2} \mathrm{O}_{5}$ ) em solução de ácido oxálico $\left(0,5 \mathrm{molL}^{-1}\right)$ sob agitação a $70^{\circ} \mathrm{C} / 12 \mathrm{~h}$, de forma que a $\%$ mássica de $\mathrm{Nb}_{2} \mathrm{O}_{5}$ fosse correspondente à $0,1 \%$. Como suporte foi utilizada a zeólita A (OXANYL CAS: 6991279-4) com $2 \mathrm{~mm}$ de diâmetro, sendo previamente seca em estufa a $100^{\circ} \mathrm{C}$ por $24 \mathrm{~h}$.

Utilizou-se o método da impregnação úmida (Schmal, 2011) para suportar os catalisadores e o procedimento consistiu em misturar a solução precursora do óxido desejado (ou as soluções no caso dos óxidos mistos) e a zeólita 3A. O solvente foi evaporado em banho termostático (Quimis) a $70^{\circ} \mathrm{C}$ sob agitação manual. Os catalisadores foram secos em estufa a $100^{\circ} \mathrm{C} / 24 \mathrm{~h}$ e calcinados em mufla (Quimis) a $400^{\circ} \mathrm{C} / 5 \mathrm{~h}$. Desta forma foram preparados quatro catalisadores: $5 \% \mathrm{ZnO} / \mathrm{A}, 10 \% \mathrm{ZnO} / \mathrm{A}, 0,1 \% \mathrm{Fe}_{2} \mathrm{O}_{3}-5 \% \mathrm{ZnO} / \mathrm{A}$ e $0,1 \% \mathrm{Nb}_{2} \mathrm{O}_{5}-5 \% \mathrm{ZnO} / \mathrm{A}$.

\subsection{Caracterização dos Fotocatalisadores}

Os fotocatalisadores preparados e a zeólita A foram caracterizados por difração de raios X (DRX) em difratômetro de raios X Shimadzu, modelo XRD 6000, tubo de CuKa em modo $2 \theta$, velocidade $2 \% \mathrm{~min}$, intensidade de $2000 \mathrm{cps}$, do Complexo de Apoio à Pesquisa da UEM, por espectroscopia fotoacústica em equipamento do Departamento de Física da UEM, por espectroscopia de absorção no infravermelho (FTIR) em espectrofotômetro Perkin Elmer FT-IR/NIR Spectrometer Frontier, a partir de pastilhas de $\mathrm{KBr}$ de 450 a $4000 \mathrm{~cm}^{-1}$ e ponto de carga zero $\left(\mathrm{pH}_{\mathrm{PCZ}}\right)$, sendo que o procedimento consistiu em misturar $0,1 \mathrm{~g}$ do fotocatalisador com $50 \mathrm{~mL}$ de solução de $\mathrm{NaCl}\left(0,01 \mathrm{molL}^{-1}\right)$ ajustada em diferentes condições de $\mathrm{pH}$ inicial (2 a 12) e manter sob agitação a temperatura ambiente por $24 \mathrm{~h}$. $\mathrm{O} \mathrm{pH}$ de equilíbrio foi determinado e o $\mathrm{pH}_{\mathrm{PCZ}}$ foi identificado quando o $\mathrm{pH}_{\text {final }}$ é igual ao $\mathrm{pH}_{\text {inicial }}$.

\subsection{Efluente}

O efluente real da etapa de tingimento da pele do peixe foi coletado ao final do processo de tingimento, processo desenvolvido nos laboratórios do Grupo de Estudo e Manejo na Aquicultura (GEMAQ-Unioeste). Este efluente possuía a coloração verde devido à 


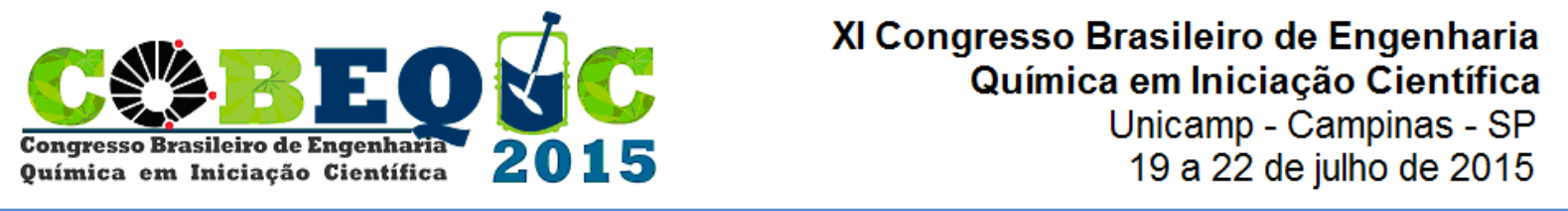

presença do corante Acid Green $16\left(\mathrm{C}_{31} \mathrm{H}_{33} \mathrm{~N}_{2} \mathrm{NaO}_{6} \mathrm{~S}_{2}\right)$ da KROMÁTICA, e ácido fórmico, utilizado para fixação da cor.

\subsection{Testes Fotocatalíticos}

Os testes fotocatalíticos foram realizados irradiando $500 \mathrm{~mL}$ do efluente (diluído 1:10), juntamente com os catalisadores e a zeólita A nas concentrações 1 e $2 \mathrm{gL}^{-1}$. Os testes tiveram duração de $3 \mathrm{~h}$ e alíquotas foram coletadas em intervalos regulares. A temperatura foi monitorada em todas as coletas, além da avaliação da descoloração por espectrofotometria UV-Vis (Shimadzu Modelo UV-1800). As amostras iniciais e finais foram submetidas a análises de $\mathrm{pH}$, turbidez e condutividade. A fotólise foi efetuada para critério de comparação.

\section{RESULTADOS E DISCUSSÃO}

\subsection{Caracterização dos Fotocatalisadores}

Os catalisadores e a zeólita A foram caracterizados por FTIR e os espectros de FTIR são apresentados na Figura 1 e pode-se observar a presença de $\mathrm{ZnO}$ pela alteração das bandas a 680 e $1380 \mathrm{~cm}^{-1}$ em comparação ao suporte e a do $\mathrm{Nb}_{2} \mathrm{O}_{5}$, a $1210 \mathrm{~cm}^{-1}$.

Figura 1 - Espectro FTIR dos fotocatalisadores suportados e da zeólita A.
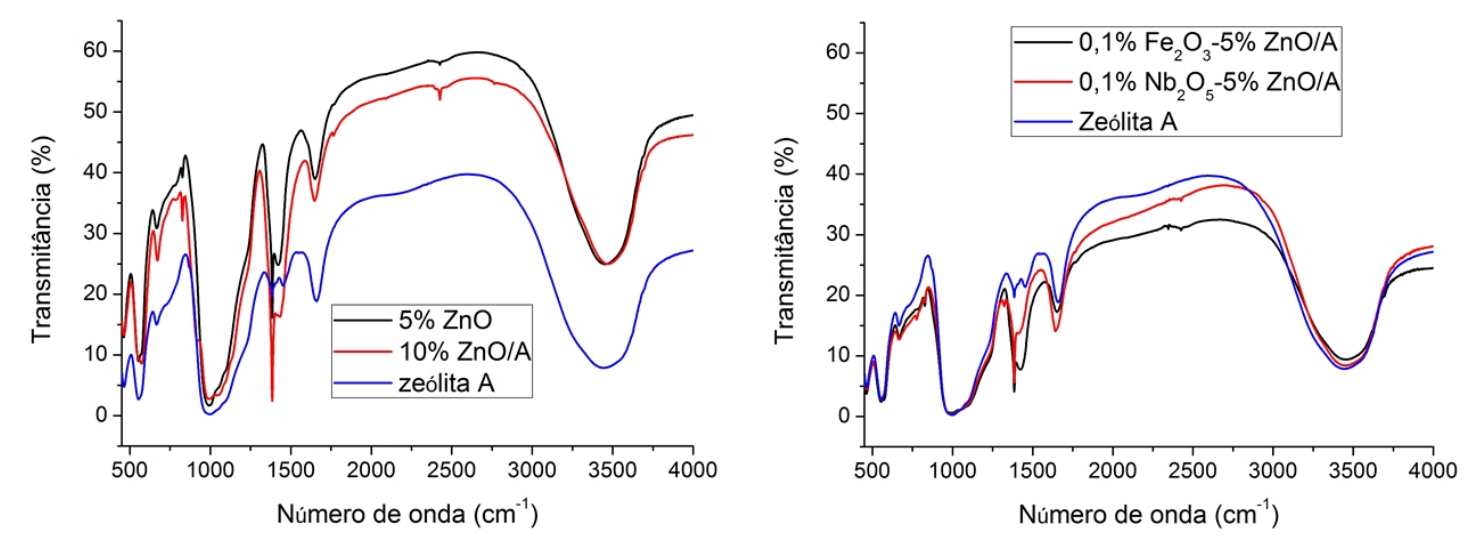

Os resultados de DRX e de fotoacústica podem ser visualizados na Figura 2. A cristalinidade da zeólita A foi mantida após o processo de impregnação. Identificou-se os picos do $\mathrm{ZnO}\left(2 \theta=37^{\circ}\right.$ e $\left.58^{\circ}\right)$ com estrutura hexagonal zincite (Marques, 2005) e do $\mathrm{Nb}_{2} \mathrm{O}_{5}$ $\left(2 \theta=39^{\circ}\right)$ com estrutura hexagonal (Marques, 2005), porém os picos do $\mathrm{Fe}_{2} \mathrm{O}_{3}$ não foram observados, possivelmente por este apresentar partículas dispersas inferiores a $20 \mathrm{~nm}$ (Figura 2.a). Na Figura 2.b, observa-se um aumento na intensidade do sinal fotoacústico com a impregnação do $\mathrm{ZnO}$ e dos óxidos mistos.

A partir dos valores de intensidade do sinal acústico foi possível calcular a energia de band gap $\left(\mathrm{E}_{\mathrm{g}}\right)$ a partir da função de Kubelka-Munk. Os valores da $\mathrm{E}_{\mathrm{g}}$ podem ser observados na Tabela 1. Os catalisadores de $\mathrm{ZnO}$ apresentaram máxima absorção em $397 \mathrm{~nm}(3,12 \mathrm{eV})$, enquanto que para os mistos, foi a $400 \mathrm{~nm}(3,10 \mathrm{eV})$. Na Tabela 1 também pode ser visualizado os valores de $\mathrm{pH}_{\mathrm{PCZ}}$ dos catalisadores. Segundo Fungaro e Borrely (2012), 
zeólitas sintetizadas a partir de cinzas de carvão possuem $\mathrm{pH}_{\mathrm{PCZ}}$ na faixa de 8 a 10. Aqui, a zeólita comercial apresentou $\mathrm{pH}_{\mathrm{PCZ}}$ de 8,7 . Além disso, verifica-se que a presença de óxido puro ou mistos impregnados na zeólita reduziu o $\mathrm{pH}_{\mathrm{PCZ}}(6,2-6,5)$.

Figura 2 - Difratogramas dos fotocatalisadores e do suporte (a) e análise por espectroscopia fotoacústica (b).

(a)
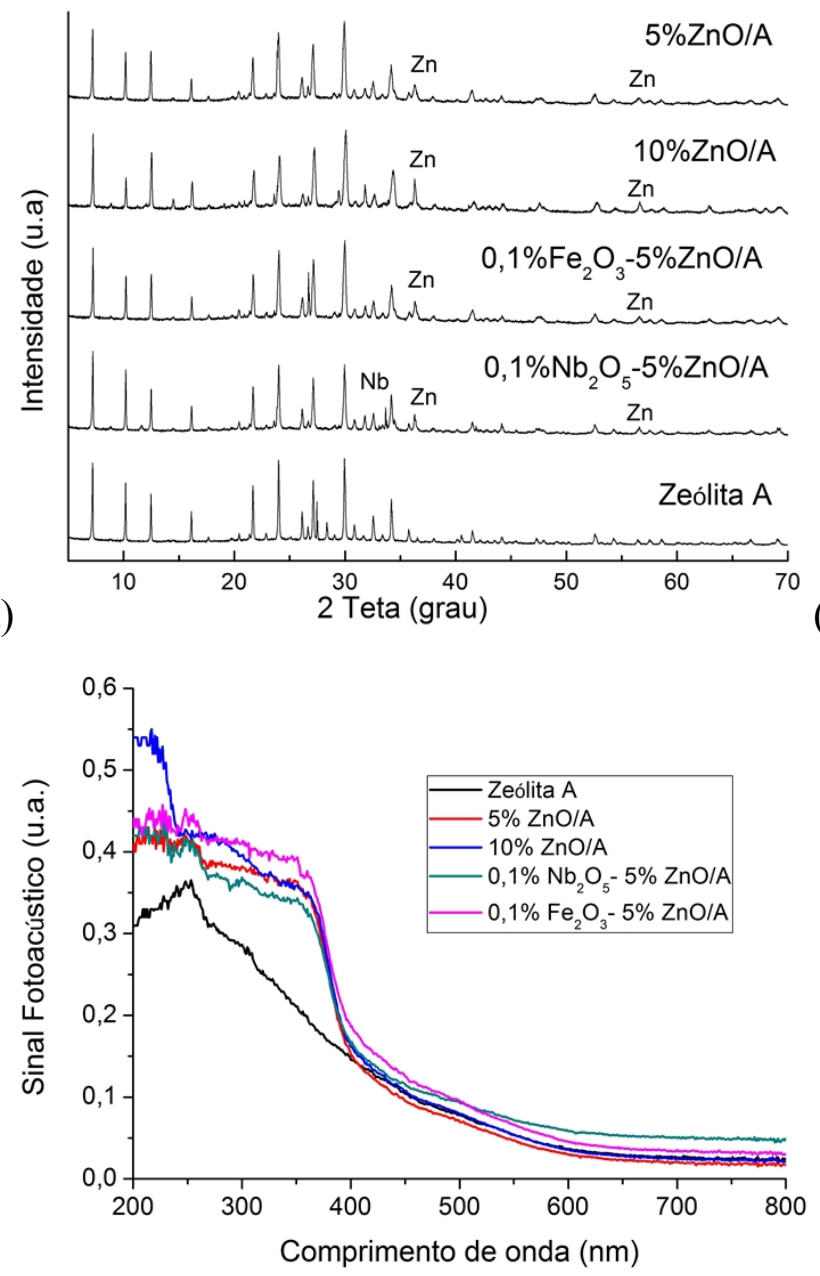

Tabela 1 - Valores de energia de band gap e $\mathrm{pH}_{\mathrm{PCZ}}$ para os fotocatalisadores.

\begin{tabular}{cccccc}
\hline Catalisador & $5 \% \mathrm{ZnO} / \mathrm{A}$ & $10 \% \mathrm{ZnO} / \mathrm{A}$ & $\begin{array}{c}0,1 \% \mathrm{Fe}_{2} \mathrm{O}_{3^{-}} \\
5 \% \mathrm{ZnO} / \mathrm{A}\end{array}$ & $\begin{array}{c}0,1 \% \mathrm{Nb}_{2} \mathrm{O}_{5^{-}} \\
5 \% \mathrm{ZnO} / \mathrm{A}\end{array}$ & Zeólita A \\
\hline $\mathrm{E}_{\mathrm{g}}(\mathrm{eV})$ & 3,12 & 3,12 & 3,10 & 3,10 & 3,23 \\
\hline $\mathrm{pH} \mathrm{PCZ}_{\mathrm{P}}$ & 6,2 & 6,5 & 6,3 & 6,3 & 8,7 \\
\hline
\end{tabular}

\subsection{Testes fotocatalíticos}

O efluente foi caracterizado e apresentou as seguintes características iniciais: $\mathrm{pH}$ 6, condutividade de $562 \mu \mathrm{Scm}^{-1}$ e turbidez de $35,8 \mathrm{NTU}$. Este efluente foi tratado por fotocatálise e os resultados encontram-se na Tabela 2 e na Figura 3. 
Tabela 2 - Resultados obtidos após os testes fotocatalíticos.

\begin{tabular}{ccccc}
\hline Condição & $\begin{array}{c}\text { Massa } \\
\left(\mathrm{gL}^{-1}\right)\end{array}$ & $\begin{array}{c}\text { Aumento da } \\
\text { Condutividade }(\%)\end{array}$ & $\begin{array}{c}\text { Redução da } \\
\text { Turbidez }(\%)\end{array}$ & Descoloração(\%) \\
\hline Fotólise & 0 & 12,3 & 34,5 & 9,8 \\
Zeólita A & 1 & 25,2 & 33,9 & 11,0 \\
& 2 & 41,5 & 43,2 & 16,5 \\
$5 \% \mathrm{ZnO} / \mathrm{A}$ & 1 & 20,4 & 43,2 & 8,4 \\
& 2 & 24,4 & 46,9 & 12,6 \\
$10 \% \mathrm{ZnO} / \mathrm{A}$ & 1 & 28,9 & 59,7 & 9,9 \\
$0,1 \% \mathrm{Fe}_{2} \mathrm{O}_{3^{-}}$ & 2 & 39,3 & 46,1 & 12,6 \\
$5 \% \mathrm{ZnO}^{-} \mathrm{A}$ & 2 & 22,3 & 44,6 & 11,2 \\
$0,1 \% \mathrm{Nb}_{2} \mathrm{O}_{5^{-}}$ & 1 & 30,4 & 24,2 & 7,6 \\
$5 \% \mathrm{ZnO} / \mathrm{A}$ & 2 & 24,1 & 48,3 & 9,7 \\
\hline
\end{tabular}

Figura 3 - Descoloração em função do tempo para fotocatalisadores a $1 \mathrm{gL}^{-1}(\mathrm{~A})$ e $2 \mathrm{gL}^{-1}$ (B).
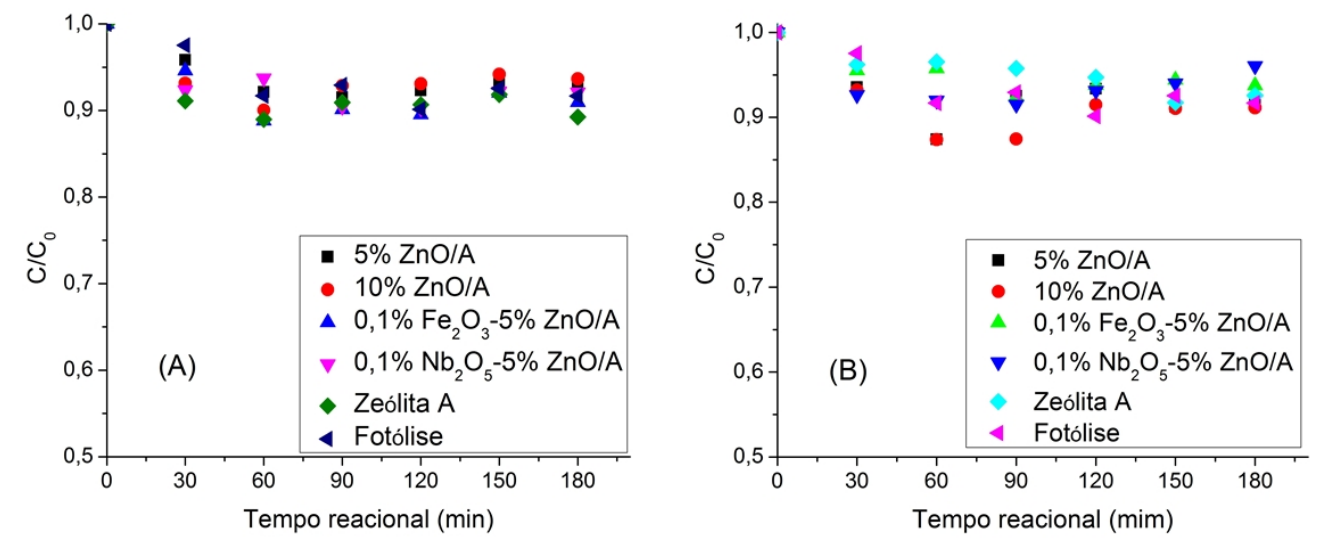

Observa-se na Tabela 2 que, em relação ao aumento da condutividade, fotólise não foi eficiente, o aumento da massa de catalisador e do teor de $\mathrm{ZnO}$ teve efeito positivo em todos os testes, e que a mistura dos óxidos apresentou efeito sinérgico positivo em comparação ao $5 \% \mathrm{ZnO} / \mathrm{A}$. A maior redução de turbidez foi obtida com $1 \mathrm{gL}^{-1}$ de $10 \% \mathrm{ZnO} / \mathrm{A}$. A descoloração deste efluente não foi significativa, ficando em torno de $12 \%$ para 5 e $10 \% \mathrm{ZnO} / \mathrm{A}$ (Tabela 2 e Figura 3). O melhor resultado foi obtido com a zeólita a pura (16,5\%). Tal comportamento pode ser explicado pelo fato dos catalisadores apresentarem ponto de carga nula próximo ao pH do efluente, enquanto que o da zeólita foi maior (Tabela 1), o que facilitaria a interação entre o efluente e a zeólita. Além disso, a zeólita absorve num comprimento de onda menor (383 nm - Tabela 1) mais próximo do comprimento de onda de maior incidência da lâmpada (310-350 nm) (Brites-Nóbrega et al., 2013).

\section{CONCLUSÃO}

Os resultados obtidos evidenciam que o método de impregnação foi satisfatório. Os testes fotocatalíticos mostraram que o aumento da massa de catalisador e o aumento do teor 


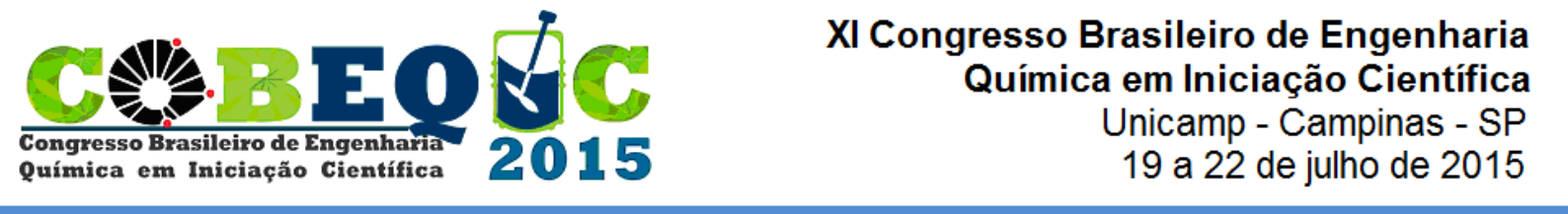

de $\mathrm{ZnO}$ apresentaram efeito positivo, e que a mistura dos óxidos apresentou efeito sinérgico positivo em comparação do catalisador $5 \% \mathrm{ZnO} / \mathrm{A}$, aumentando a eficiência do processo em termos de aumento da condutividade.

\section{AGRADECIMENTOS}

Os autores agradecem à Fundação Araucária pelo suporte financeiro.

\section{REFERÊNCIAS BIBLIOGÁFICAS}

BRITES-NÓBREGA, F. F.; POLO, A. N. B.; BENEDETTI, A. M.; LEÃO, M. M. D.; SANTANA, V. S.; FERNANDES-MACHADO, N. R. C. Evaluation of photocatalytic activities of supported catalysts on $\mathrm{NaX}$ zeolite or activated charcoal. J. Hazard. Mater., v. 263P, p. 61-66, 2013.

FELTRIN, C. W. Síntese e propriedades do ZnO: correlação entre propriedades estruturais e atividade fotocatalítica. Dissertação de mestrado, Universidade Federal do Rio Grande do Sul, 2010.

FUNGARO, D. A.; BORRELY S. I. Synthesis and characterization of zeolite from coal ashes modified by cationic surfactant. Cerâmica, v. 58, p. 77-83, 2012.

LIMA G. G. C.; LIMA C. A. P.; VIEIRA F. F.; SILVA E. M.; Estudo comparativo da aplicação de nanopartículas de $\mathrm{TiO}_{2}$ e $\mathrm{ZnO}$ na descoloração fotocatalítica de uma solução de corante empregando radiação UV artificial. REMAP, v. 9, n. 1, p. 22-27, 2014.

MALUF, M. Curtimento ecológico de peles de peixes, Gráfica e Editora Jofel, Toledo - PR, 2010.

MARIN, P.; BORBA, C. E.; MÓDENES, A. N,; ESPINOZA-QUIÑONES, F. R.; OLIVEIRA, S. P. D.; KROUMOV, A. D. Determination of the mass transfer limiting step of dye adsorption onto commercial adsorbent by using mathematical models. Environ. Technol., v. 35, n. 17-20, p. 2356-64, 2014.

MARQUES R. G. Decomposição Fotocatalitica de Gasolina Sintética e Comercial. Dissertação de mestrado, Universidade Estadual de Maringá, 2005.

MERG, J. C.; ROSSET, F.; PENHA, F. G.; PERGHER, S. B. C. Incorporação de dióxido de titânio em zeólitas para emprego em fotocatálise. Quim. Nova, v. 33, n. 7, p.1525-1528, 2010 .

PETERNELA, P.; SANTANA, V. S. Preparação e Avaliação da Atividade Fotocatalítica dos Catalisadores $\mathrm{Nb}_{2} \mathrm{O}_{5}$-ZnO/Zeólita A na Degradação do Corante Reativo Azul 5G. Anais do III Encontro Paranaense de Engenharia e Ciência, 2013.

REBOUÇAS, P. M.; SILVA, M. C.; BARBOSA FILHO, J. A. D.; NASCIMENTO, C. B. Gerando renda no semiárido cearense: sistema artesanal de curtimento de pele de Tilápia no município de Pentecoste. Anais do VII Congresso Brasileiro de Agroecologia, 2011.

SCHMAL, M. Catálise Heterogênea, Editora Synergia, Rio de Janeiro, 2011. 